Journal of Computer Science 8 (5): 752-760, 2012

ISSN 1549-3636

(C) 2012 Science Publications

\title{
The Impact of Radio Propagation Models on Ad Hoc Networks Performances
}

\author{
${ }^{1}$ Rhattoy, A. and ${ }^{2}$ A. Zatni \\ ${ }^{1}$ Department of Computer, \\ Modeling Systems and Telecommunications Research Group, \\ Moulay Ismail University, Higher School of Technology, \\ B.P. 3103, 50000, Toulal, Meknes, Morocco \\ ${ }^{2}$ Department of Computer, Optronics Laboratory, Ibnou Zohr University, \\ Higher School of Technology, B. P. 33/S, 80000, Agadir, Morocco
}

\begin{abstract}
Problem statement: Wireless networks are characterized by a dynamic topology triggered by the nodes mobility. Thus, the wireless multi-hops connection and the channel do not have determinist behaviour such as: Interference or multiple paths. Moreover, the nodes' invisibility makes the wireless channel difficult to detect. This wireless networks' behaviour should be scrutinized. Approach: In our study, we mainly focus on radio propagation models by observing the evolution of the routing layer's performances in terms of the characteristics of the physical layer. Results: For this purpose, we first examine and then display the simulation findings of the impact of different radio propagation models on the performance of ad hoc networks. To fully understand how these various radio models influence the networks performance, we have compared the performances of several routing protocols (DSR, AODV and DSDV) for each propagation model. In order to reach credible results, we focused on the notion of nodes' speed and the number of connections by using the well known network simulator NS-2. Conclusion: To conclude, the simulation findings are to be taken as a strong reference on the three routing protocols' behaviour; however, it shouldn't be considered as an exact representation of its behaviour and real environment because of several simulation constraints such as: the dimension of movement field of mobile nodes, the traffic type and the simulation timing.
\end{abstract}

Key words:MANET, ad-hoc networks, routing protocols, DSR, DSDV, AODV, fading, propagation model, economic impact

\section{INTRODUCTION}

Before using a wireless network or installing the stations of a cellular network, we have to determine the radio waves' targeted coverage. The targeted radio coverage has a crucial economic impact because it determines the equipment to be utilized. In other words, the bigger the coverage is the fewer antennas are required to cover the region or to reach a grand area. Besides, the radio coverage depends on several parameters such as the emission power. However, the environment where the waves spread and the utilized frequency also play a crucial role. The radio propagation waves are controlled by strict rules, mainly when there are obstacles between the transmitter and the receiver (Zang and Rowe, 2007; Kaya et al., 2009). Among the changes a wave may undergo, we can cite: reflection, diffraction, diffusion and absorption (Fig. 1).

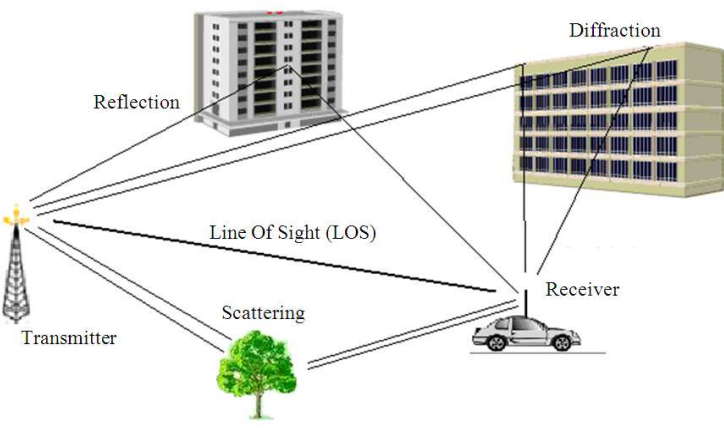

Fig. 1: The different physical phenomena disturbing radio signal propagation

The rest of this study is organized as follows. We give the radio propagation models types. Then we discuss of routing protocols concepts in ad hoc networks. In addition, we declare the methodologies of

Corresponding Author: Rhattoy, A., Department of Computer, Modeling, Systems and Telecommunications Research Group, Moulay Ismail University, Higher School of Technology, Agouray Road, Km 5, B. P. 3103,

50000, Toulal, Meknes, Morocco 
simulation. Finally, we investigate the impact of radio propagation models on the performances of routing protocols in ad hoc networks and we present our conclusions.

Radio propagation models: In a propagation model, we use a set of mathematical models which are supposed to provide an increasing precision. Propagation radio models are three types: path loss, shadowing and fading. The first type can be expressed as the power loss during the signal propagation in the free space. The second type is characterized by fixed obstacles on the path of the radio signal propagation. The third category is the fading which is composed of multiple propagation distances, the fast movements of transmitters and receivers units and finally the reflectors (Eltahir, 2007).

Free space model: The free space model assumes that in the ideal propagation condition between the transmitter and the receiver, there is only one clear Line Of Slight (LOS) path. The following equation calculate the received signal power in a free space Eq. 1:

$P_{r}(d)=\frac{P_{t} G_{t} G_{r} \lambda^{2}}{(4 \pi)^{2} d^{2} L}$

where, $P_{t}$ is the power transmission (in watts), $G_{t}$ and $G_{r}$ are the antenna gains of the transmitter and receiver respectively. $\mathrm{L}$ is the system loss factor. $\lambda$ is the wave length and $\mathrm{d}$ is the distance between the transmitter and the receiver (Singh and Kapang, 2011)

Two-ray ground model: The free space model mentioned above states that there is only one single direct path. In fact, the signal reaches the receiver through multiple paths (due to reflection, refraction and scattering). The two-path model attempts to account for this phenomenon. In other words, the model advocates that the signal attains the receiver via true paths: a lineof-slight path and a path through which the reflected wave is received (Singh and Kapang, 2011). In the twopath model, the received power is represented by Eq. 2 :

$$
P_{r}(d)=\frac{P_{t} G_{t} G_{r} h_{t}^{2} h_{r}^{2}}{d^{4} L}
$$

where, $h_{t}$ and $h_{r}$ are the heights of the transmitter and receiver respectively. Nonetheless, for short distances, the two-ray model does not give accurate results because of in oscillation caused by the constructive and destructive combination of the two rays. The propagation model in the free space is instead, still used where $d$ is small. Hence, in this model, we calculate $d_{c}$ as a cross-over distance. When $\mathrm{d}<\mathrm{d}_{c}$, we use the first equation, but when $d<d_{c}$, the second equation is used. At the cross-over distance, Eq. 1 and 2 give similar results. Consequently, $d_{c}$ can be calculated as Eq. 3:

$\mathrm{d}_{\mathrm{c}}=\frac{4 \pi \mathrm{h}_{\mathrm{t}} \mathrm{h}_{\mathrm{r}}}{\lambda}$

Shadowing model: Both the free space and the two-ray models predict the received power in terms of the distance. They also represent a communication area as an ideal circle. In fact, the received power at a given distance varies randomly because of multi-path propagation effects, known as fading effects. Thus, the two aforementioned models predict the mean received power at distance $d$. The shadowing model is twofold (Singh and Kapang, 2011). The first model is the path loss model represented by $\mathrm{P}_{\mathrm{r}}(\mathrm{d})$. It employs a close in distance $\mathrm{d}_{0}$ as follows Eq. 4:

$$
\frac{\mathrm{P}_{\mathrm{r}}\left(\mathrm{d}_{0}\right)}{\mathrm{P}_{\mathrm{r}}(\mathrm{d})}=\left(\frac{\mathrm{d}}{\mathrm{d}_{0}}\right)^{\beta}
$$

$\beta$ is called the path loss exponent and is often empirically determined by filed measurement. Equation 3 implies that $\beta=2$ in free space propagation. The Table 1 gives typical values of $\beta$ (Fall, 2001).

Langer values of $\beta$ correspond to more obstructions and thus faster decrease in average received power as distance becomes larger. From Eq. 4, we have:

$$
\left[\overline{\frac{\mathrm{P}_{\mathrm{r}}(\mathrm{d})}{\mathrm{P}_{\mathrm{r}}\left(\mathrm{d}_{0}\right)}}\right]_{\mathrm{dB}}=-10 \beta \log \left(\frac{\mathrm{d}}{\mathrm{d}_{0}}\right)
$$

The second part of the shadowing model reflects the variations of received power at certain distance (Eq. 5 ). It is a log-normal random variable. The overall model is represented by Eq. 6:

$$
\left[\frac{\overline{\mathrm{P}_{\mathrm{r}}(\mathrm{d})}}{\mathrm{P}_{\mathrm{r}}\left(\mathrm{d}_{0}\right)}\right]_{\mathrm{dB}}=-10 \beta \log \left(\frac{\mathrm{d}}{\mathrm{d}_{0}}\right)+\mathrm{X}_{\mathrm{dB}}
$$

where, $\mathrm{X}_{\mathrm{dB}}$ is Gaussian random variable with zero mean and standard deviation $\sigma_{\mathrm{dB}}$

$\sigma_{\mathrm{dB}}$ is called shadowing deviation and also obtained through measurement in the real environment. Table 2 displays some typical values of $\sigma_{\mathrm{dB}}$. This equation is also labelled a log-normal shadowing model. 
Table 1: Some Typical values of path loss $\beta$

\begin{tabular}{lll}
\hline & Environment & $\beta$ \\
\hline Outdoor & Free space & 2 \\
& Shadowed urban area & $2.7-5$ \\
In building & Line-of-sight & $1.6-1.8$ \\
& Obstructed & $4-6$ \\
\hline
\end{tabular}

Table 2: Typical values of shadowing deviation odB

Environment

Outdoor

Office, hard partition

Office, soft partition

Factory, line-of-sight

Factory, obstructed

Small-scale fading model: Rayleigh and rice: This fading model depicts the rapid fluctuations of the received signal due to multipath fading. This fading phenomenon is generated by the interference of at least two types of transmitted signals to the receiver with slight time intervals (Amjad and Stocker, 2010). The outcome may vary according to fluctuations and to different phases in terms of multiple factors such as: delay between waves, the intensity and the signal band width. Hence, the system performance may be attenuated by the fading. However, there are several techniques that help stopping this fading. The signal fading were monitored according to a statistical law wherein the most frequently used distribution is Raleigh's (Carvalho et al., 2004). The transmitted signal is, thus, conditioned by the following phenomena: reflection, scattering and diffusion. Thanks to these three phenomena, the transmitted power may reach the hidden areas despite the lack of direct visibility (NLOS) between the transmitter and receiver. Consequently, the amount of the received signal has a density of Rayleigh Eq. 7:

$f(x)= \begin{cases}\frac{2 x}{P} \exp \left(-\frac{x^{2}}{P}\right), & \text { pour } 0 \leq x \leq \infty \\ 0 & , \text { pour } x<0\end{cases}$

where, $\mathrm{P}$ is the average received power. In case where there is a direct path (LOS) between the transmitter and receiver, the signal no longer obeys to Rayleigh's law but to Rice's. The probability density of Rice is represented by Eq. 8:

$$
f(x)=\left\{\begin{array}{l}
\frac{2 x(K+1)}{P} \exp \left(-K-\frac{(K+1) x^{2}}{P}\right) I_{0} \\
\left(2 x \sqrt{\frac{K(K+1)}{P}}\right), \text { pour } 0 \leq x \leq \infty \\
0, \text { pour } x<0
\end{array}\right.
$$

Where:

$\mathrm{K}=$ The ratio of the power received in the direct line and in the path

$\mathrm{P} \quad=$ The average power received

$\mathrm{I}_{0}(\mathrm{x})=$ The zero-order Bessel function de fined by Eq. 9:

$I_{0}(x)=\frac{1}{2 \pi} \int_{0}^{2 \pi} \exp (-x \cos \theta) d \theta$

The density of Rice is reduced to the density of Rayleigh in the case of an absence of a direct path which means that $\mathrm{K}=0$ and thus $\mathrm{I}_{0}(\mathrm{x})=1$.

Nakagami model: This distribution encompasses several other distributions as particular cases. To describe Rayleigh distribution, we assumed that the transmitted signals are similar and their phases are approximate. Nakagami model is more realistic in that it allows similarly to the signals to be approximate. Since we have used the same labels as in Rayleigh and Rice cases, we have $r=\left|\sum r_{i} e^{j \theta_{i}}\right|$. The probability density of Nakagami related to $r$ is represented by Eq. 10:

$\mathrm{P}_{\mathrm{r}}(\mathrm{r})=\frac{2 \mathrm{~m}^{\mathrm{m}} \mathrm{r}^{2 \mathrm{~m}-1}}{\Gamma(\mathrm{m}) \Omega^{\mathrm{m}}} \exp \left(-\frac{\mathrm{mr}^{2}}{\Omega}\right), \mathrm{r} \geq 0$

where, $\Gamma(\mathrm{m})$ is gamma function, $\Omega=\left(\mathrm{r}^{2}\right)$ and $\mathrm{m}=\{\mathrm{E}$ $\left.\left(\mathrm{r}^{2}\right)\right\}^{2} / \operatorname{var}\left(\mathrm{r}^{2}\right)$ with the constraint $\mathrm{m} \geq 1 / 2$. Nakagami model is a general distribution of fading which is reduced to Rayleigh's distribution for $\mathrm{m}=1$ and to unilateral Gaussian model for $m=1 / 2$. Besides, it represents pretty much rice model and it is closer to certain conditions in the lognormal distribution.

Ad hoc routing protocols: Ad hoc routing protocols are based on fundamental principles of routing such as: Inundation (flooding), the distance Vector, the routing to the source and the state of the site. According to the way routes are created and maintained during the data delivery, the routing protocols can be characterised into two categories: proactive and reactive (Feeney, 1999). Among the tested protocols in this study, only DSDV is proactive and the others (DSR and AODV) are all reactive. Proactive protocols update route information periodically, whereas reactive protocols establish routes only when needed. Here is a summary of the routing protocols assessed in this study.

Dynamic Source Routing (DSR): During the discovery process of routing, a source node generates a route-request packet which needs a new route to a certain destination. The route request is connected 
through the network until it reaches some nodes with a route to destination. A reply packet containing all information of intermediate nodes is sent back to the source. The sent packets contain a list of all nodes through which they have to transit. This list can be huge in a network with a big diameter. The nodes do not need the routing table. There are two DSR basic operations: the route discovery and the route maintenance. In order to cut down the expenses and the frequency of the route discovery, every single node keeps track of the paths thanks to reply packets. These paths are used until they become useless (Khatri and Rajput, 2010).

Ad-hoc On-Demand Distance Vector protocol (AODV): AODV has a way for route request close to that of DSR. However, AODV does not perform a routing to the source. Every single node on the path refers to a point towards its neighbour from which it receives a reply. When a transit node needs broadcasts a route request to a neighbour, it also stores the node identifier in the routing table from which the first reply is received. To check the links state, AODV uses control messages (Hello) between direct neighbours. Besides, AODV utilizes a sequence number to avoid a round trip and to ensure using the most recent routes (Alfawaer and Hua, 2007).

DSDV protocol: The algorithm Dynamic destination Sequenced Distance Vector (DSDV) (Gupta and Saket, 2011; Ramesh et al., 2010) has been constructed for mobile networks. Each mobile station keeps a routing table which contains all possible destinations, number of hops to reach the destination, Sequence Number (SN) associated with the node destination to distinguish the new routes of the old a ones and avoid the formation of round trip routing. The table updating is periodically transmitted across the network so as to sustain the information consistency and thus generates an important traffic.

\section{MATERIALS AND METHODS}

Methodology: In this study, on one hand we study the impact of different propagation models in order to analyse the environment effect on the ad hoc networks' performance. On the other hand, we have compared several routing protocols performances (DSR, AODV and DSDV) according to every propagation models. In order to obtain valid results, we have inserted the notion of the nodes speed and the number of connections. The assessment is twofold: First, we diversified the nodes' speed. Second, we altered the number of connections.
Scenario 1: So as to analyse the ad hoc routing protocols' behaviour, we selected traffic sources with a constant output (CBR) related to UDP protocol. The packet emission rate is settled at 8 packets per second with a maximal speed variation of nodes. Ten speed values were considered: 1, 2, 3, 4, 5, 6, 7, 8, 9 and $10 \mathrm{~m}$ $\mathrm{sec}^{-1}$. The assessed protocols are: AODV, DSR and DSDV. These three are available in 2.34 of $n s-2$. The propagation models under study are: the free space, the two-Ray ground, Rice's and Nakagami's models. The simulation span is of $200 \mathrm{sec}$. The data packet size is 512 octets. The mobile nodes utilize the random waypoint mobility model (Geetha and Gopinath, 2008). The Mobil nodes move within a square dimension area $670 \times 670 \mathrm{~m}$. At the moment, we limit the number of sources in 10 and we analyse the impact of the nodes' speed.

Scenario 2: The number of sources may be another parameter that can be altered so as to look at the different routing protocols' performance. In this part, we display the impact of the traffic load on the routing protocols. For this reason, we have varied a number of connections. Six cases were considered: 5, 10, 15, 20, 25 and 30 connections. For the time being, let's limit the nodes' maximal speed at $10 \mathrm{~m} \mathrm{sec}^{-1}$ while the other parameters are similar to those in the first case.

Performance indicators: Because of the length chosen in this study, we have selected just three performance indicators in order to study the routing protocols performances. They are outlined as follows: Packet delivery fraction, end average to end delay and the throughput.

Packet Delivery Fraction (PDF): This is the ratio of total number of CBR packets successfully received by the destination nodes to the number of CBR packets sent by the source nodes throughout the simulation:

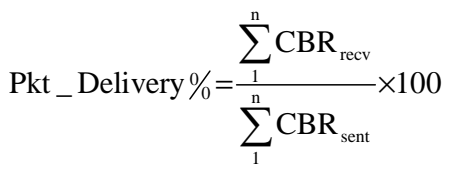

This estimation gives us an idea of how successful the protocol is in delivering packets to the application layer. A high value of PDF indicates that most of the packets are being delivered to the higher layers and it is a good indicator of the protocol performance.

Average End-To-End Delay (AE2E Delay): This is defined as the average delay in transmission of a packet between two nodes and is calculated as follows: 
Avg_End_to_End_delay $=\frac{\sum_{1}^{\mathrm{n}}\left(\mathrm{CBR}_{\text {sent_Time }}-\mathrm{CBR}_{\text {recv_Time }}\right)}{\sum_{1}^{\mathrm{n}} \mathrm{CBR}_{\text {recv }}}$

A higher value of end-to-end delay means that the network is congested and hence the routing protocol does not perform well. It depends on the physical characteristics of a link and the delay of treatment.

Throughput: The throughput data reflects the effective network capacity. It is computed by dividing the message size with the time it took to arrive at its destination. It is measured considering the hops performed by each packet.

\section{RESULTS AND DISCUSSION}

Simulation findings: In this part, we display the study findings about the impact of the nodes' maximal speed and the traffic load on the routing protocols; according to the three aforementioned performance indicators: packets Delivery fraction, Throughput and average end to end delay.

Scenario 1: The results corresponding to the PDF, AE2E Delay and throughput are shown in Fig. 2-4 respectively.

Packet delivery fraction: In Fig. 2, we notice that the packet delivery fraction varies slightly according to the speed increase. Consequently, the links are relatively steady and weaker with a weak speed. AODV and DSR offer more packets than DSDV. Besides, when the nodes' speed increases, the packet delivery decreases a bit in case of DSDV. Hence, the main reason for the packet loss is mobility, congestion and the wireless channel characteristics.

Meanwhile, we notice that the free-space and the two-ray ground deliver more packets than the other models such as; first Rice, second Rayleigh third Nakagami and finally the shadowing. Rice's model performance operates according to straight sight and employs the free-space for long distance prediction. Whereas, the shadowing bad performance is due to the low intensity of the signal caused by the obstacles. This results in the packet loss on weak links, displays wrongly the links disconnection and leads to the interruption and thus the dire need to set up a new itinerary. DSR reacts badly to the use of shadowing and Nakagami models because the two models create a very dynamic topology in our simulations. Since DSR relies heavily on stored paths, it is more inclined to utilize information about lost paths. Consequently, this generates high packet routing fraction and low packet delivery.

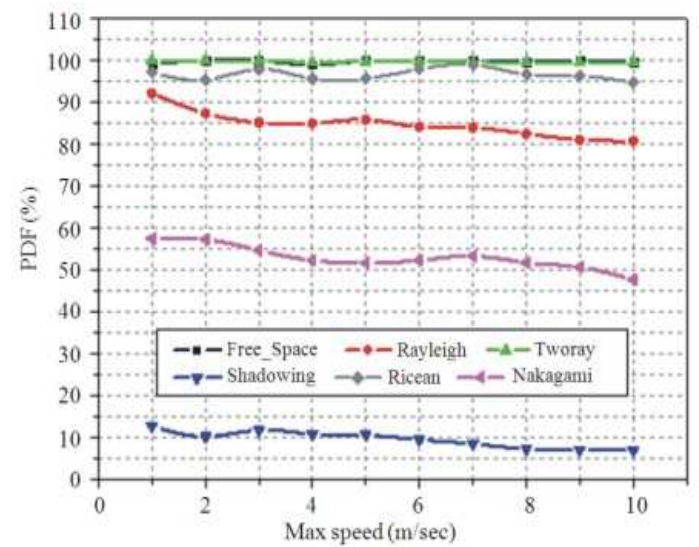

(a)

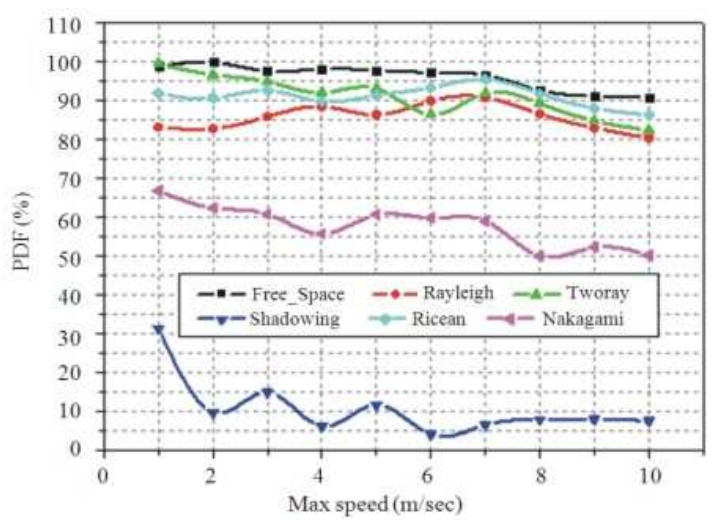

(b)

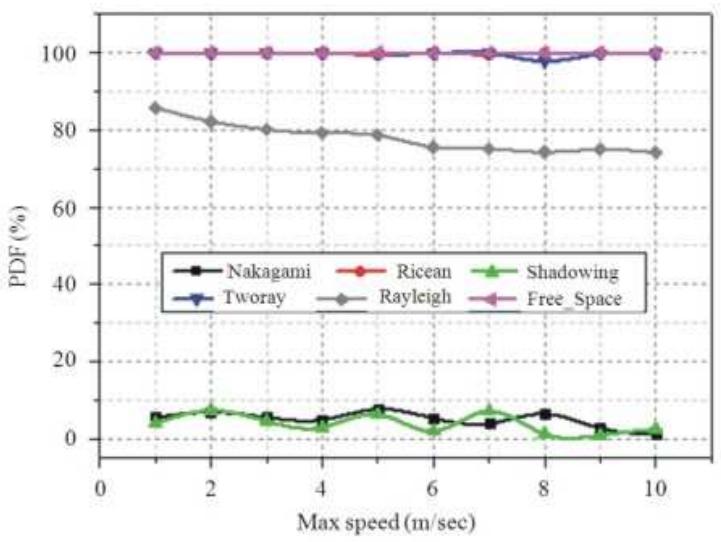

(c)

Fig. 2: (a) AODV- PDF (b) DSDV-PDF (c) DSR-PDF versus Speed 


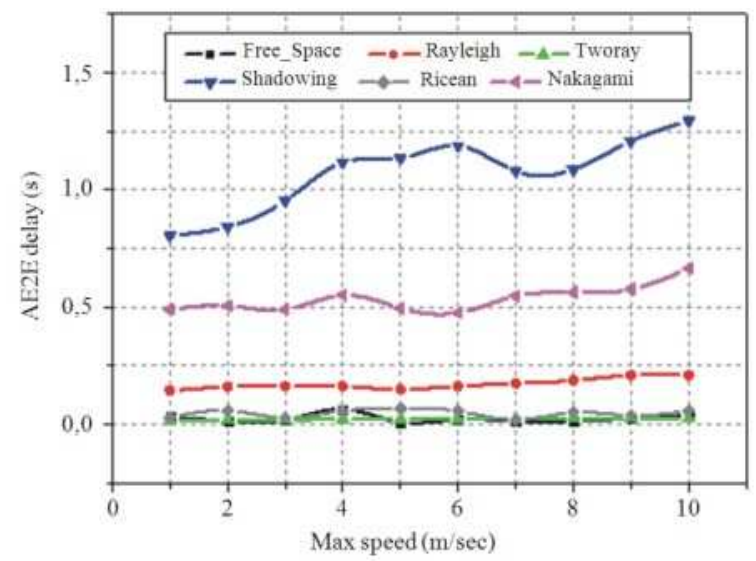

(a)

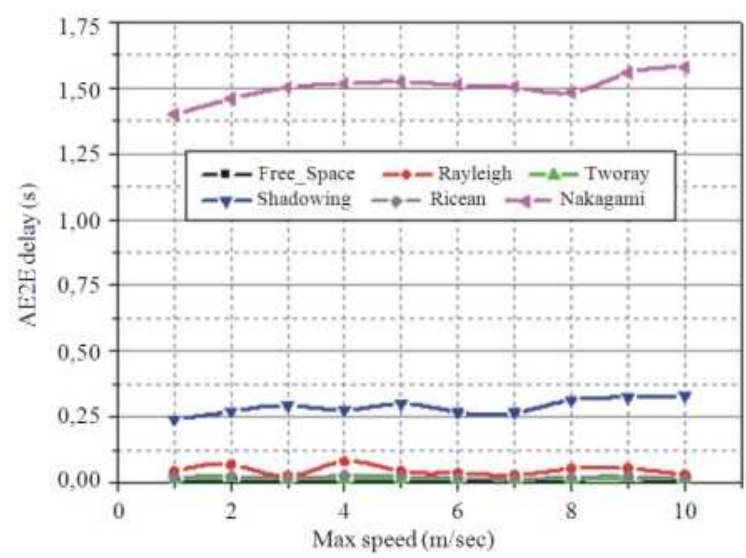

(b)

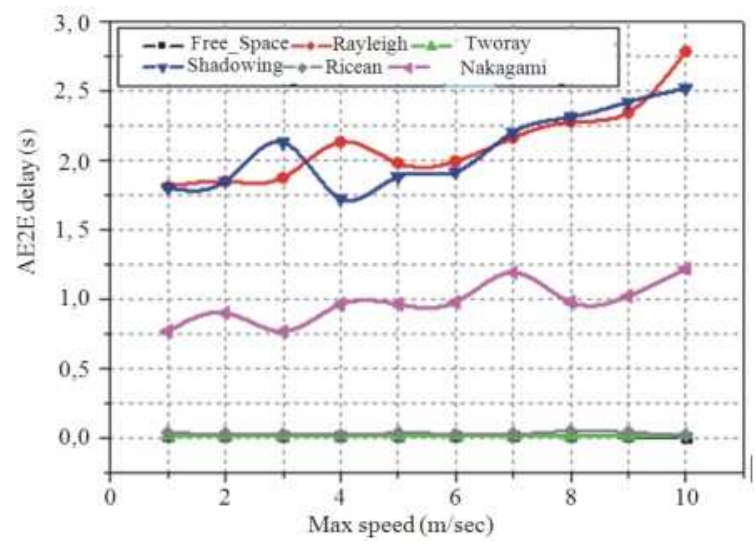

(c)

Fig. 3: (a) AODV-AE2E Delay (b) DSDV-AE2E Delay (c) DSR-AE2E Delay versus Speed

Average end-to-end delay: Figure 3, displays that DSR has more system timing than AODV and DSDV because in DSR, the intermediate nodes are allowed to reply through stored paths in their memories, which are unfortunately, often invalid. Hence, the transmitted data packets will be deleted once they reach their broken links. In addition, the data packets in DSR undergo extra delays during the communication interfaces' waiting because of the frequent retransmissions. This latency causes the packets death (their deletion). Similarly to PDF, we notice that the free-space and the two-ray ground endure less delay than the other models, followed by first Rice, second Rayleigh third Nakagami and finally the shadowing model. The weak performance of shadowing and Nakagami stems from the fact that when we observe the slope indicating the un-mentioned collisions' rate, we realize that the phenomenon is accounted for. The nodes' mobility has an influence on every single parameter; in other words, it influences mainly the end-to-end delay.

Throughput: As mentioned in part PDF, the higher the received packets rate. As we expected, the throughput decreases slightly when the speed increases because it has to find the path for more routing traffic delivery. Therefore, the channel will be less used for the data transfer to as to reduce the useful throughput. Like AODV, in case of DSDV, the throughput decreases as the speed increases (Fig. 4).

Scenario 2: The results corresponding to the PDF, AE2E Delay and Throughput are shown in Fig. 5-7 respectively.

Packet delivery fraction: Figure 5, displays, different routing protocols performances in terms of the number of connections. The charts also display that if the number of connections increases, the delivery fraction value tends to decrease for all models. Thus, there is network congestion.

In this scenario, DSDV is less preferment than AODV and DSR because their PDF are over $99 \%$ in so far as it reaches 10 connections. However, when we increase the number of connections in PDF, DSR should be compared to AODV.

Average end-to-end delay: In Figure 6, as expected, the delay is higher for non direct-sight propagation models (NLOS). Moreover, as there are more deliveries, the average delay also increases. Consequently, the packets have to wait more in a stand by position. In term of delays, we can observe that DSDV and AODV are more efficient than DSR. We also notice that delays for the two protocols increase rapidly according to the number of connections because of the high traffic congestion in some areas of the ad hoc networks. DSDV, this is accounted for by its use of priority criteria where in the protocol packet is given priority. 


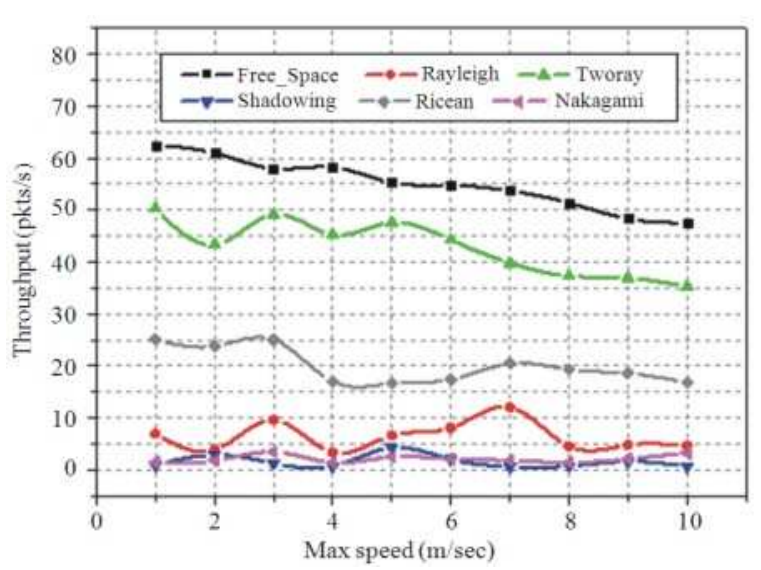

(a)

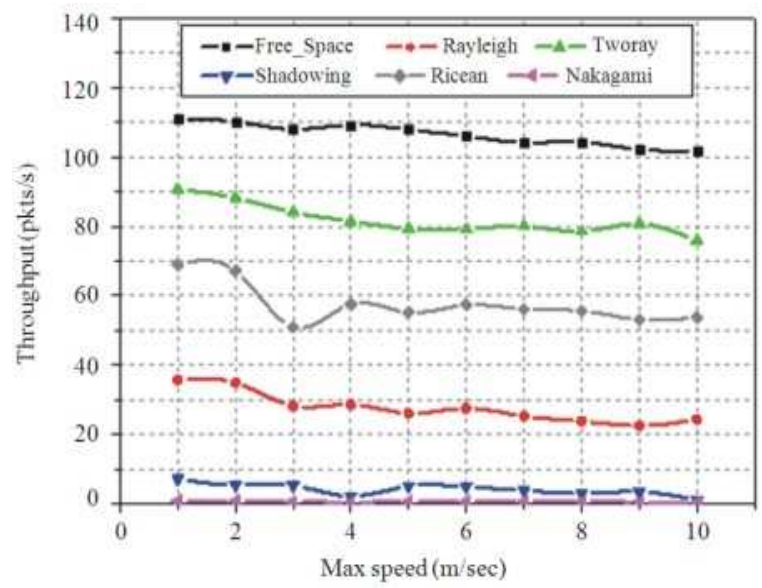

(b)

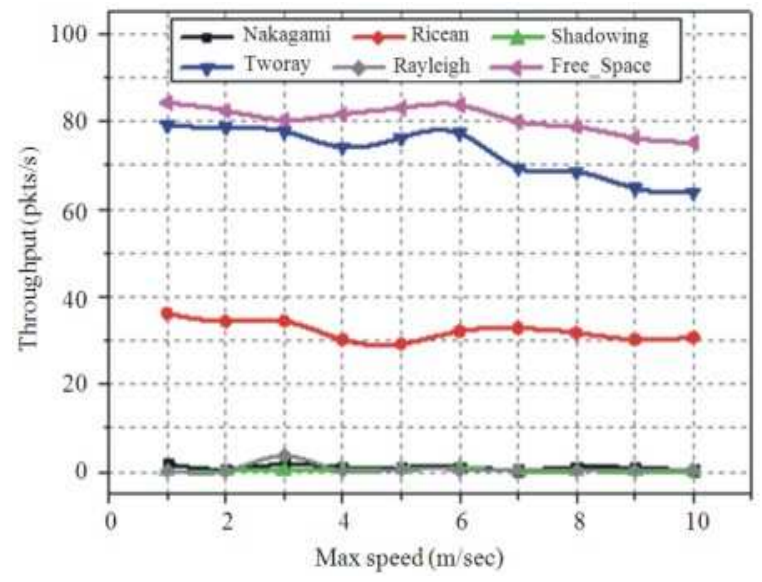

(c)

Fig. 4: (a) AODV-Throughput (b) DSDVThroughput (c) DSR-Throughput versus speed

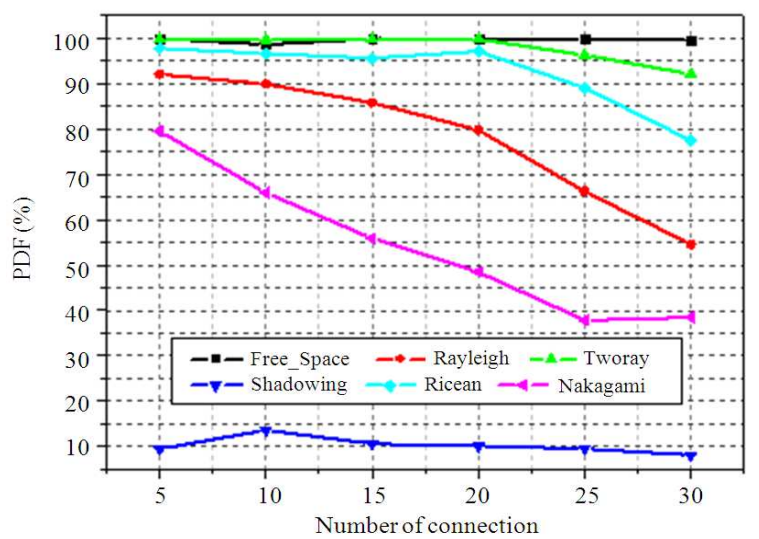

(a)

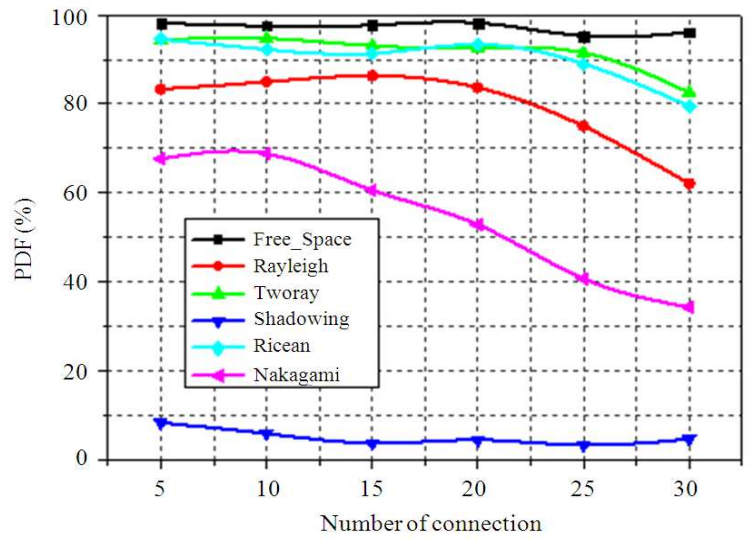

(b)

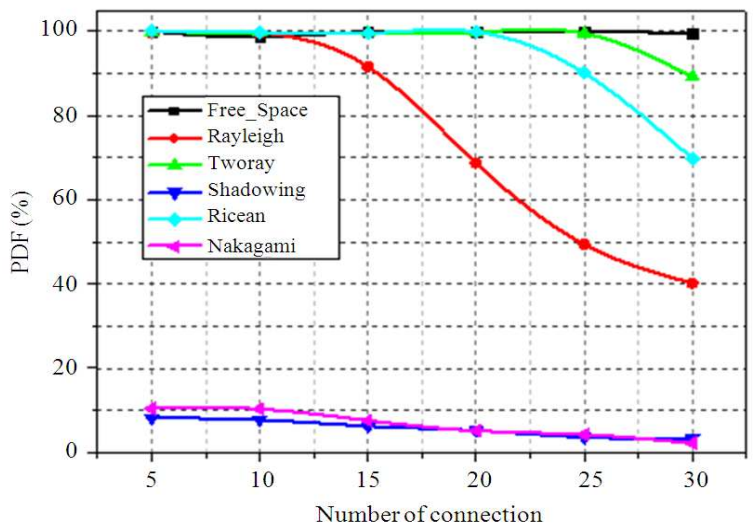

(c)

Fig. 5: (a) AODV-PDF (b) DSDV- PDF (c) DSR-PDF versus speed number of connections

Hence, a protocol packet is always treated prior to any data packet even if it arrives later. On the other hand DSDV does not distinguish between the protocol packets and the data ones during the waiting phase. Instead all packets are treated according to their arrival ranking. 


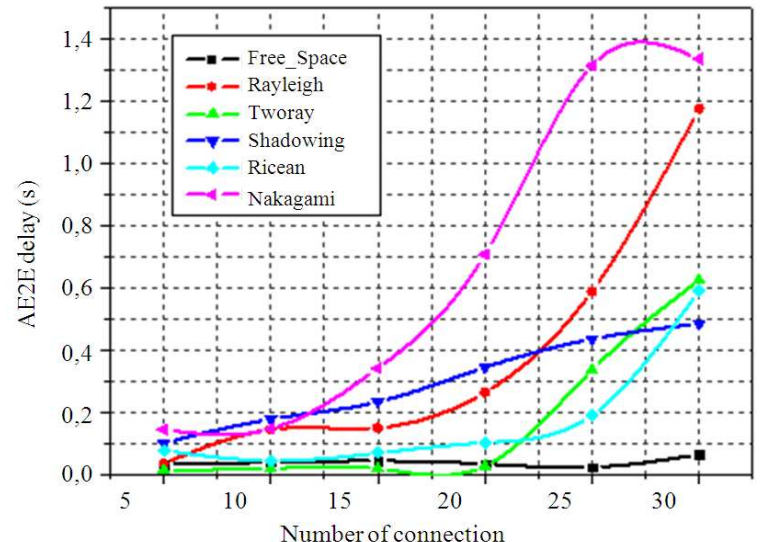

(a)

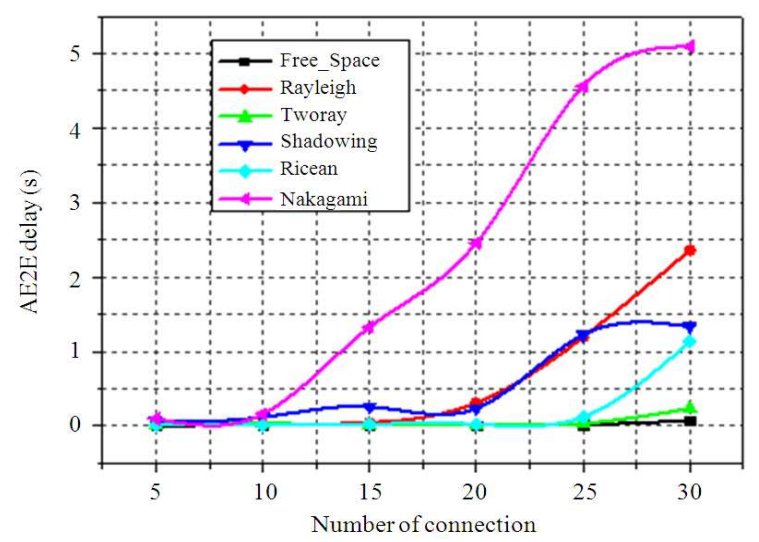

(b)

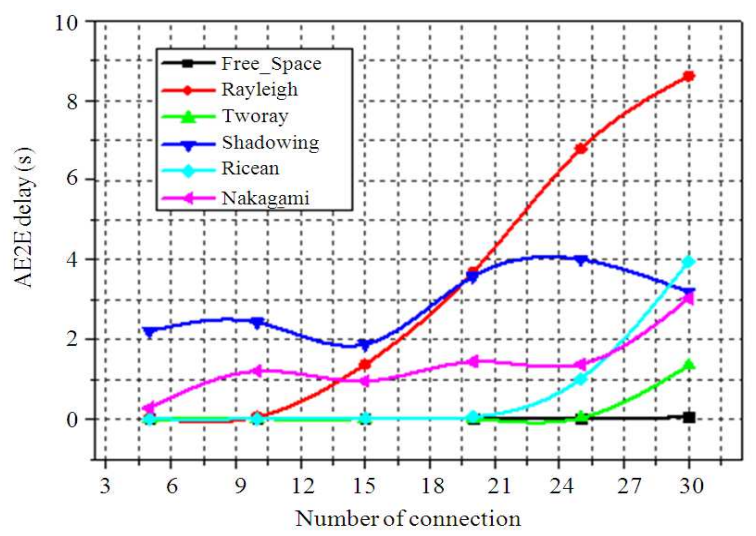

(c)

Fig. 6: (a) AODV-AE2E Delay (b) DSDV-AE2E Delay (c) DSR-AE2E Delay versus number of connections

Throughput: In Figure 7, we notice that the throughput diminishes significantly with an increase of the traffic load. DSDV protocol is steadier than AODV for the increasing number of connections.

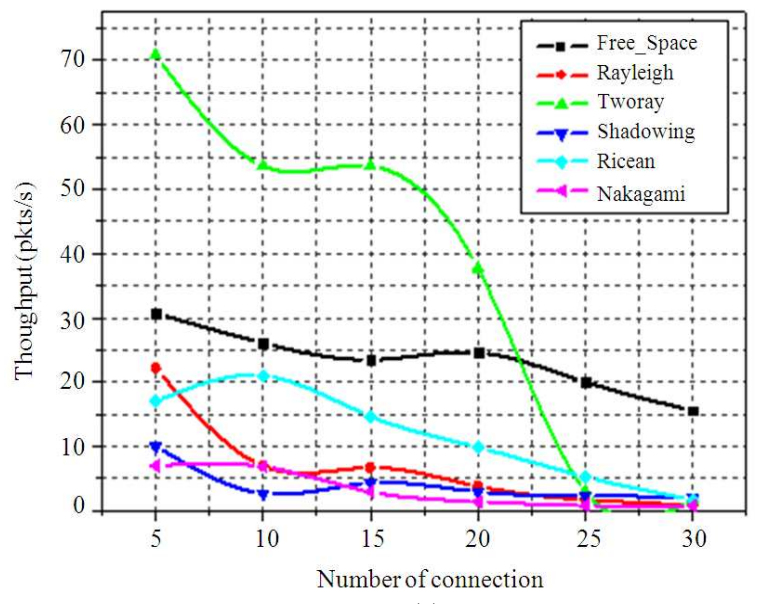

(a)

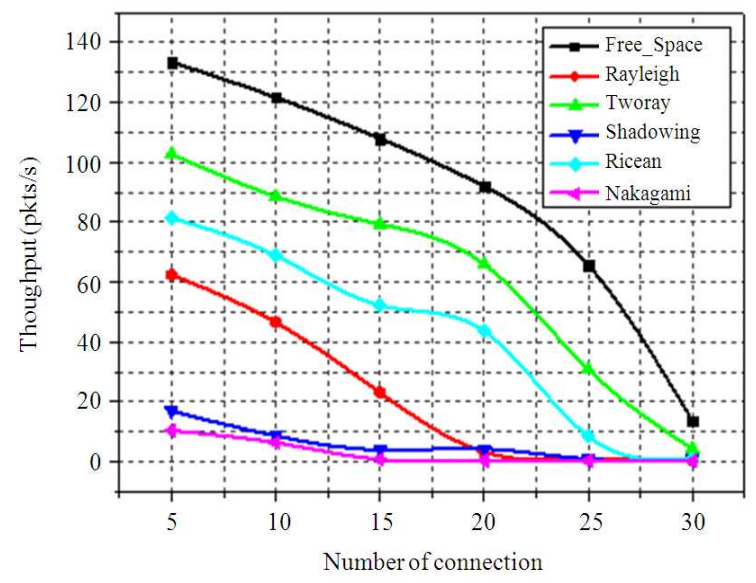

(b)

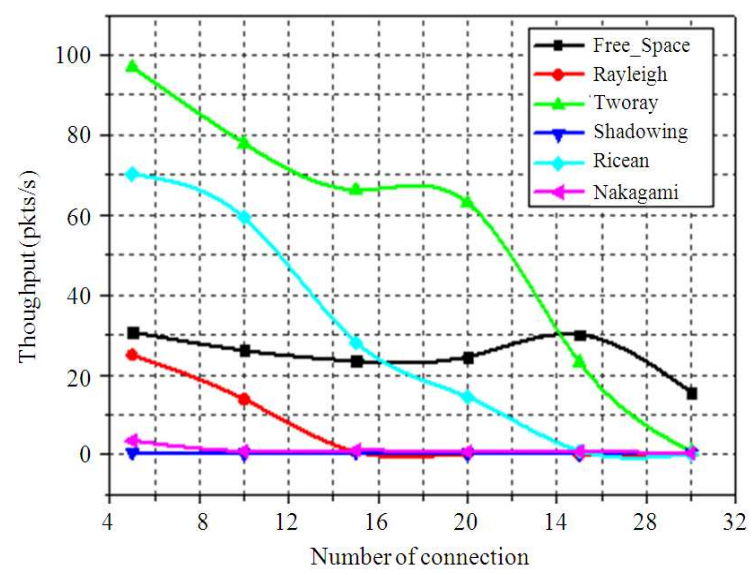

(c)

Fig. 7: (a) AODV-Throughput (b) DSDV-Throughput (c) DSR-Throughput versus number of connections 


\section{CONCLUSION}

Conclusions and perspectives: In this article, we study the impact of different radio propagation models on the performance of ad hoc networks. According to the simulation findings, we may state that the choice of the propagation models has a great impact on the routing protocol's performance. In this respect, we have identified both the determinist and the statistic modelizations. The simulation findings have revealed that the different propagation models have a considerable impact on the performance of the ad hoc mobile network. The latter decreases rapidly when the fading models, mainly Ricean, Rayleigh, Shadowing and Nakagami have been taken into consideration. The main reasons of their deterioration are the outcome of the big variation in the received intensity signal.

According to the results to the routing protocols' performance, we find out that there is no preferable protocol among the others all scenarios and the assessing criteria. On the other hand, no matter how the nodes' speed is, the DSDV is more efficiency in terms of the delay because of its proactive features. However, its activity sharing is very weak, which influences the network stability and thus becomes weak; whereas, the activity concentration is high. AODV and DSR have the best performances in terms of the delivery packet fraction. DSR uses the cash memory for the route discovery. This factor decreases the delay performances which may be due to the excessive use of cash memory and the inability to delete the old routes. Nonetheless, it seems that the use of cash memory enables DSR to maintain a weak overload.

To conclude, the simulation findings are to be taken as a strong reference on the three routing protocols' behaviour; however, it shouldn't be considered as an exact representation of its behaviour and real environment because of several simulation constraints such as: the dimension of movement field of mobile nodes, the traffic type and the simulation timing. In the forthcoming studies, we will look at the routing protocols' behaviours in the multi-channel environment and/or multi-networks in order to determine the key parameters that have an impact on the protocols' choice. Besides, we will try to develop new protocols or alter the existing ones.

\section{REFERENCES}

Amjad, K. and A.J. Stocker, 2010. Impact of slow and fast channel fading and mobility on the performance of AODV in ad-hoc networks. Proceedings of the Loughborough Antennas and Propagation Conference (LAPC), Nov. 8-9, IEEE Xplore Press, Loughborough, pp: 509-512. DOI: 10.1109/LAPC.2010.5666192
Alfawaer, Z.M. and G.W. Hua, 2007. Utilization of AODV in wireless ad hoc networks. J. Comput. Sci., 3: 218-222. DOI: 10.3844/jcssp.2007.218.222

Carvalho, M.M. and J.J. Garcia-Luna-Aceves, 2004. Modeling single-hop wireless networks under Rician fading channels. Proceedings of the IEEE Wireless Communications and Networking Conference, Mar. 21-25, IEEE Xplore Press, pp: 219-224. DOI: 10.1109/WCNC.2004.1311546

Eltahir, I.K., 2007. The impact of different radio propagation models for Mobile Ad hoc NETworks (MANET) in urban area environment. Proceedings of The 2nd International Conference on Wireless Broadband and Ultra Wideband Communications, Aug. 27-30, IEEE Xplore Press, Sydney, NSW, pp: 30-30. DOI: 10.1109/AUSWIRELESS.2007.80

Fall, K., 2001. The ns manual. National Institute of Informatics.

Feeney, L.M., 1999. A taxonomy for routing protocols in mobile ad hoc networks. SICS Report.

Geetha, J and G. Gopinath, 2008. Performance Comparison of Two On-demand Routing Protocols for ad-hoc networks based on random way point mobility model. Am. J. Applied Sci., 5: 659-664. DOI: 10.3844/ajassp.2008.659.664

Gupta, S.K. and R.K. Saket, 2011. Performance metric comparison of AODV and DSDV routing protocols in manets using ns-2. IJRRAS, 7: 339-350. www.arpapress.com/Volumes/Vol7Issue3/IJRRAS _7_3_15.pdf

Kaya, A.O., L.J. Greenstein and W. Trappe, 2009. Characterizing indoor wireless channels via ray tracing combined with stochastic modeling. IEEE Trans. Wireless Commun., 8: 4165-4175. DOI: 10.1109/TWC.2009.080785

Khatri, P. and M. Rajput, 2010. Performance study of ad-hoc reactive routing protocols. J. Comput. Sci., 6: 1159-1163. DOI: 10.3844/jcssp.2010.1159.1163

Ramesh, V., P. Subbaiah, N.K. Rao and M.J. Raju, 2010. Performance comparison and analysis of DSDV and AODV for MANET. Int. J. Comput. Sci. Eng., 2: 183-188.

Singh, P.K. and L. Kapang, 2011. Comparative study of radio propagation and mobility models in vehicular adhoc network. Int. J. Comput. Appl., 16: 37-42. DOI: $10.5120 / 2031-2600$

Zang, L.F. and G.B. Rowe, 2007. Improved modelling for mobile Ad-hoc networks. Elect. Lett., 43: 11561156. DOI: 10.1049/el:20071530 